\title{
Algorithm of High-Precision Tracking of Secondary Sources Optical Radiation and its Efficiency
}

\author{
Alexander A. Dontsov*, \\ Yuri L. Koziratsky and Dmitry V. Prochorov \\ Military Education and Research Centre of Military-Air Forces \\ «Military-Air Academy \\ Named After Professor N.E. Zhukovsky and Yu.A. Gagarin» \\ 54a Starykh Bolshevikov Str., Voronezh, 394064, Russia
}

Received 16.02.2016, received in revised form 13.03.2016, accepted 21.08.2016

With use of methods of the theory of a filtration of existential signals the algorithm of highprecision tracking of moving object according to the image of its secondary source of optical radiation (an engine torch) is synthesized. It is shown that due to introduction of procedure of an assessment of equivalent radius of the image of a torch of the engine the algorithm provides steady tracking coordinates of moving object with a mistake no more than two elements of permission.

Keywords: high-precision maintenance, secondary source of optical radiation, nonlinear filtration of existential signals.

Citation: Dontsov A.A., Koziratsky Yu.L., Prochorov D.V. Algorithm of high-precision tracking of secondary sources optical radiation and its efficiency, J. Sib. Fed. Univ. Eng. technol., 2016, 9(8), 1144-1152. DOI: 10.17516/1999-494X-2016-9-8-11441152 .

(C) Siberian Federal University. All rights reserved

* Corresponding author E-mail address: addoncov1@mail.ru 


\section{Алгоритм высокоточного сопровождения вторичных источников оптического излучения и его эффективность}

А.А. Донцов, Ю.Л. Козирацкий, Д.В. Прохоров Военный учебно-научный центр Военно-воздушных сил «Военно-воздушная академия имени профессора Н.Е. Жуковского и Ю.А. Гагарина» Россия, 394064, Воронеж, ул. Старых Большевиков, 54 а

С использованием методов теории фильтрации пространственно-временных сигналов синтезирован алгоритм высокоточного сопровождения движущегося объекта по изображению его вторичного источника оптического излучения (факела двигателя). Показано, что за счет введения процедуры оценки эквивалентного радиуса изображения факела двигателя алгоритм обеспечивает устойчивое слежение за координатами движущегося объекта с ошибкой не более двух элементов разрешения.

Ключевые слова: высокоточное сопровождение, вторичный источник оптического излучения, нелинейная фильтрация пространственно-временных сигналов.

\section{Введение}

Задача автоматического сопровождения движущихся объектов предусматривает наличие в контуре управления измерителя, предназначенного для оптимальной (квазиоптимальной) оценки текущих координат объекта по информации, заключенной в сигналах различной физической природы. В качестве источника такой информации может использоваться изображение факела двигателя управляемого боеприпаса. В этом случае управление системой сопровождения возможно с помощью матричного датчика, формирующего изображение факела двигателя как вторичного источника оптического излучения. Поскольку координаты энергетического центра изображения факела двигателя управляемого боеприпаса с достаточной точностью определяют его текущее местоположение, возникает задача разработки алгоритма определения координат движущегося объекта по изображению визируемой сцены и вторичного источника оптического излучения (факела двигателя).

\section{Постановка задачи}

Задачу синтеза алгоритма определения координат будем решать на основе использования аппарата нелинейной пространственно-временной фильтрации [1-3]. Аппроксимируем движение объекта в плоскости, перпендикулярной линии визирования матричного датчика, системой стохастических дифференциальных уравнений:

$$
\frac{\partial X(t)}{\partial t}=-\frac{X(t)}{T_{x}}+n_{x}(t) ; \quad \frac{\partial Y(t)}{\partial t}=-\frac{Y(t)}{T_{y}}+n_{y}(t),
$$

где $X(t), Y(t)$ - текущие координаты объекта по горизонтальной и вертикальной осям в картинной плоскости, рассчитываемые относительно центра изображения, определяемого по- 
ложением линии визирования цели; $T_{x}, T_{y}$ - эквивалентные постоянные времени; $n_{x}, n_{y}$ - нормальные белые шумы с нулевым математическим ожиданием и спектральными плотностями $N_{x}, N_{y}$.

Полезный сигнал (изображение факела двигателя), наблюдаемый на текущем изображении визируемой сцены и являющийся функцией интересующих нас параметров, формально представим двумерным экспоненциальным законом вида

$$
s(x, y, t)=A e^{-\frac{(x-X(t))^{2}}{2 R_{u}(t)^{2}}} e^{-\frac{(y-Y(t))^{2}}{2 R_{u}(t)^{2}}},
$$

где $A$ - амплитуда наблюдаемого полезного сигнала; $R_{u}(t)=R_{u 0}+\Delta R_{u}(t)$ - эквивалентный радиус изображения факела двигателя; $\Delta R_{u}(t)$ - приращение эквивалентного радиуса к априорному значению $R_{u 0}$.

Дополним систему уравнений (1) дифференциальным уравнением для значений приращения эквивалентного радиуса изображения факела двигателя

$$
\frac{\partial \Delta R_{u}(t)}{\partial t}=-\frac{\Delta R_{u}(t)}{T_{R}}+n_{R}(t)
$$

где $T_{R}$ - эквивалентная постоянная времени; $n_{R}-$ нормальный белый шум с нулевым математическим ожиданием и спектральной плотностью $N_{R}$.

Представим пространственно-яркостную структуру наблюдаемого текущего изображения следующим выражением:

$$
u(x, y, t)=f(x, y)+s(x, y, t)+n(x, y, t),
$$

где $f(x, y)$ - исходное изображение сцены в отсутствие движущегося объекта; $n(x, y, t)$ - гауссовский однородный пространственно-временной шум наблюдения со спектральной плотностью $N$ и корреляционной функцией вида

$$
r(\Delta x, \Delta y, \Delta t)=N \delta(\Delta x, \Delta y) \delta(\Delta t),
$$

где $\delta(\Delta x, \Delta y), \delta(\Delta t)-\delta$-функции от приращений пространственных и временной переменных соответственно.

Теперь задача синтеза алгоритма определения координат движущегося объекта может быть сформулирована как задача совместной оценки по наблюдаемому изображению (4) переменных $X, Y, \Delta R_{u}$, изменение которых во времени определяется уравнениями (1) и (3).

\section{Синтез алгоритма высокоточного сопровождения}

Введем единообразную систему обозначений оценок переменных $\hat{X}, \hat{Y}, \Delta \hat{R}_{u}$, подлежащих определению

$$
\hat{\lambda}=\left(\hat{\lambda}_{1}, \hat{\lambda}_{2}, \hat{\lambda}_{3}\right)=\left(\hat{X}, \hat{Y}, \Delta \hat{R}_{u}\right)
$$

Тогда в соответствии с [2] в гауссовском приближении квазиоптимальные оценки переменных $\left(\hat{\lambda}_{1}, \hat{\lambda}_{2}, \hat{\lambda}_{3}\right)$ по наблюдаемому изображению $u(x, y, t)$ представляются следующими выражениями в векторно-матричной форме: 


$$
\begin{aligned}
& d \hat{\lambda} / d t=\mathbf{a}(t, \hat{\lambda})+\mathbf{R}(\mathbf{t}) \mathbf{F}_{1}(t, \hat{\lambda}), \\
& d \mathbf{R} / d t=\mathbf{N}+\mathbf{a}_{1}(t, \hat{\lambda}) \mathbf{R}(\mathbf{t})+\mathbf{R}(\mathbf{t}) \mathbf{a}_{1}^{\mathrm{T}}(t, \hat{\lambda})+\mathbf{R}(\mathbf{t}) \mathbf{F}_{2}(t, \hat{\lambda}) \mathbf{R}(\mathbf{t}),
\end{aligned}
$$

где $\mathbf{a}(t, \hat{\lambda})=\left(-\hat{\lambda}_{1} / T_{x},-\hat{\lambda}_{2} / T_{y},-\hat{\lambda}_{3} / T_{R}\right)^{\mathrm{T}} ; \mathbf{a}_{1}(t, \hat{\lambda})-$ матрица размерности $3 \times 3$ с элементами $a_{i j}=\partial a_{i}(t, \hat{\lambda}) / \partial \hat{\lambda}_{j} ; \mathbf{F}_{1}(t, \hat{\lambda})=\left(\partial F(t, \hat{\lambda}) / \partial \hat{\lambda}_{1}, \ldots, \partial F(t, \hat{\lambda}) / \partial \hat{\lambda}_{3}\right) ; \mathbf{F}_{2}(t, \hat{\lambda})-$ матрица размерности $3 \times 3$ с элементами $F_{i j}=\partial^{2} \mathbf{F}_{2}(t, \hat{\lambda}) / \partial \hat{\lambda}_{j} \partial \hat{\lambda}_{j} ; \mathbf{N}$ - диагональная матрица размерности $3 \times 3 \mathrm{c}$ элементами $N_{11}=N_{x}, N_{22}=N_{y}, N_{33}=N_{R}$;

$$
F(t, \hat{\lambda})=-\frac{1}{2 N} \iint_{M}[u(x, y, t)-(f(x, y)+s(x, y, t))]^{2} d x d y
$$

- функционал правдоподобия [3]; $M$ - область определения изображения.

\section{Определение дискриминационных характеристик алгоритма}

Рассмотрим более подробно элементы $\partial F(t, \hat{\boldsymbol{\lambda}}) / \partial \hat{\lambda}_{i}$ вектора $\mathbf{F}_{1}(t, \hat{\boldsymbol{\lambda}})$, математические ожидания которых можно интерпретировать как дискриминационные характеристики многосвязной следящей системы, устанавливающие связь между наблюдаемым текущим изображением сцены и регулирующим воздействием по соответствующей координате. По аналогии с [4-7] упростим выражение (9) для функционала правдоподобия $F(t, \hat{\lambda})$. После возведения в квадрат подинтегрального выражения, перегруппировки слагаемых и исключения из дальнейшего рассмотрения слагаемых, не зависящих от оцениваемых переменных, выражение для функционала правдоподобия может быть представлено в упрощенном виде:

$$
F(t, \hat{\lambda})=\frac{A}{N} \iint_{M} u(x, y, t) \exp \left(-\frac{\left(x-\hat{\lambda}_{1}\right)^{2}+\left(y-\hat{\lambda}_{2}\right)^{2}}{2\left(R_{u 0}+\hat{\lambda}_{3}\right)^{2}}\right) d x d y .
$$

Теперь после дифференцирования выражения (10) по $\hat{\lambda}_{1}, \hat{\lambda}_{2}, \hat{\lambda}_{3}$ элементы вектора $\mathbf{F}_{1}(t, \hat{\lambda})$ принимают следующий вид:

$$
\begin{aligned}
& \partial F(t, \hat{\lambda}) / \partial \hat{\lambda}_{1}=\frac{A}{N} \iint_{M} u(x, y, t) \frac{x-\hat{\lambda}_{1}}{\left(R_{u 0}+\hat{\lambda}_{3}\right)^{2}} \mathrm{e}^{-\frac{\left(x-\hat{\lambda}_{1}\right)^{2}+\left(y-\hat{\lambda}_{2}\right)^{2}}{2 \cdot\left(R_{u 0}+\hat{\lambda}_{3}\right)^{2}}} d x d y, \\
& \partial F(t, \hat{\lambda}) / \partial \hat{\lambda}_{2}=\frac{A}{N} \iint_{M} u(x, y, t) \frac{y-\hat{\lambda}_{2}}{\left(R_{u 0}+\hat{\lambda}_{3}\right)^{2}} \mathrm{e}^{-\frac{\left(x-\hat{\lambda}_{1}\right)^{2}+\left(y-\hat{\lambda}_{2}\right)^{2}}{2 \cdot\left(R_{u 0}+\hat{\lambda}_{3}\right)^{2}}} d x d y, \\
& \partial F(t, \hat{\lambda}) / \partial \hat{\lambda}_{3}=\frac{A}{N} \iint_{M} u(x, y, t) \frac{\left(x-\hat{\lambda}_{1}\right)^{2}+\left(y-\hat{\lambda}_{2}\right)^{2}}{\left(R_{u 0}+\hat{\lambda}_{3}\right)^{3}} \mathrm{e}^{-\frac{\left(x-\hat{\lambda}_{1}\right)^{2}+\left(y-\hat{\lambda}_{2}\right)^{2}}{2 \cdot\left(R_{u 0}+\hat{\lambda}_{3}\right)^{2}}} d x d y .
\end{aligned}
$$

На рис. 1 представлена дискриминационная характеристика системы слежения за координатами центра изображения факела двигателя в горизонтальной плоскости, рассчитанная с помощью выражения (11) и определяющая зависимость сигнала управления на выходе дискриминатора от ошибки оценивания координат $\lambda_{1}-\hat{\lambda}_{1}\left(\lambda_{1}-\right.$ истинное значение координаты объекта в горизонтальной плоскости, измеряемое в элементах разрешения (э. р.) изображения). Дискриминационная характеристика рассчитана для следующей совокупности исходных данных: 


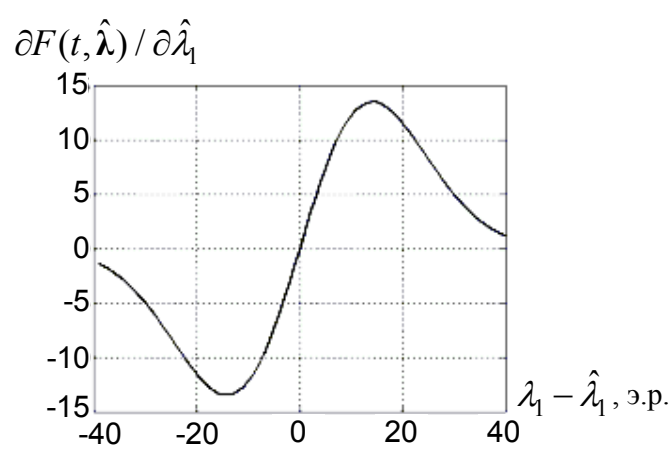

Рис. 1. Дискриминационная характеристика системы слежения за координатами

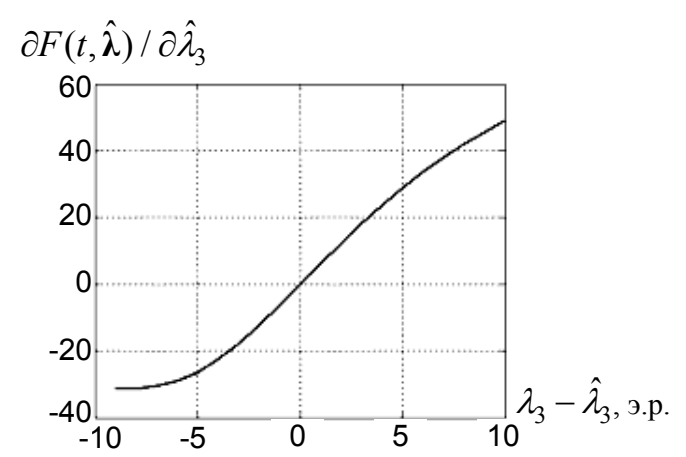

Рис. 2. Дискриминационная характеристика системы слежения за приращением радиуса

$A=1 ; \lambda_{2}-\hat{\lambda}_{2}=0$ э. р.; $R_{u 0}=10$ э. р.; $\lambda_{3}=0$ э. р. В качестве изображения фона $f(x, y)$ использовалось синтезированное методами компьютерной графики гауссовское случайное поле с заданными параметрами закона распределения яркостей элементов изображения $\left(m_{\phi}=0, \sigma_{\phi}=0,1-\right.$ математическое ожидание и СКО яркости элементов изображения фона) и изотропными корреляционными свойствами.

Из рис. 2 представлена дискриминационная характеристика системы слежения за приращением эквивалентного радиуса изображения факела двигателя, рассчитанная с помощью выражения (13) ( $\lambda_{3}$ - истинное значение приращения эквивалентного радиуса), при условии, что $A=1 ; \lambda_{1}-\hat{\lambda}_{1}=0$ э. р.; $\lambda_{2}-\hat{\lambda}_{2}=0$ э.р.; $R_{u 0}=10$ э. р. Из рис. 1 и 2 видно, что дискриминационные характеристики в окрестности истинных значений параметров линейны.

\section{Уточнение дифференциальных уравнений квазиоптимальной фильтрации}

Система дифференциальных уравнений квазиоптимальной фильтрации, полученная с помощью (7), (11) - (13) в предположении о некоррелированности ошибок оценки рассматриваемых параметров $\left(R_{i j}=0\right.$, при $\left.i \neq j\right)$ и соответствующая выражениям $(1)-(4)$, имеет вид

$$
\begin{gathered}
\frac{\partial \hat{\lambda}_{1}}{\partial t}=-\frac{\hat{\lambda}_{1}}{T_{x}}+\frac{A R_{11}}{N} \iint_{M} u(x, y, t) \frac{x-\hat{\lambda}_{1}}{\left(R_{u 0}+\hat{\lambda}_{3}\right)^{2}} \mathrm{e}^{-\frac{\left(x-\hat{\lambda}_{1}\right)^{2}+\left(y-\hat{\lambda}_{2}\right)^{2}}{2\left(R_{u 0}+\hat{\lambda}_{3}\right)^{2}}} d x d y, \\
\frac{\partial \hat{\lambda}_{2}}{\partial t}=-\frac{\hat{\lambda}_{2}}{T_{y}}+\frac{A R_{22}}{N} \iint_{M} u(x, y, t) \frac{y-\hat{\lambda}_{2}}{\left(R_{u 0}+\hat{\lambda}_{3}\right)^{2}} \mathrm{e}^{-\frac{\left(x-\hat{\lambda}_{1}\right)^{2}+\left(y-\hat{\lambda}_{2}\right)^{2}}{2\left(R_{u 0}+\hat{\lambda}_{3}\right)^{2}}} d x d y, \\
\frac{\partial \hat{\lambda}_{3}}{\partial t}=-\frac{\hat{\lambda}_{3}}{T_{R}}+\frac{A R_{33}}{N} \iint_{M} u(x, y, t) \frac{\left(x-\hat{\lambda}_{1}\right)^{2}+\left(y-\hat{\lambda}_{2}\right)^{2}}{\left(R_{u 0}+\hat{\lambda}_{3}\right)^{3}} \mathrm{e}^{-\frac{\left(x-\hat{\lambda}_{1}\right)^{2}+\left(y-\hat{\lambda}_{2}\right)^{2}}{2\left(R_{u 0}+\hat{\lambda}_{3}\right)^{2}}} d x d y .
\end{gathered}
$$

Общая структура синтезированного алгоритма определения координат движущегося объекта, соответствующая (14), приведена на рис. 3. Она представляет собой схему многоканальной следящей системы, осуществляющей слежение за центром изображения факела двигателя. При этом замыкание петель обратной связи производится по переменным $\hat{\lambda}_{1}$ и $\hat{\lambda}_{2}$ 


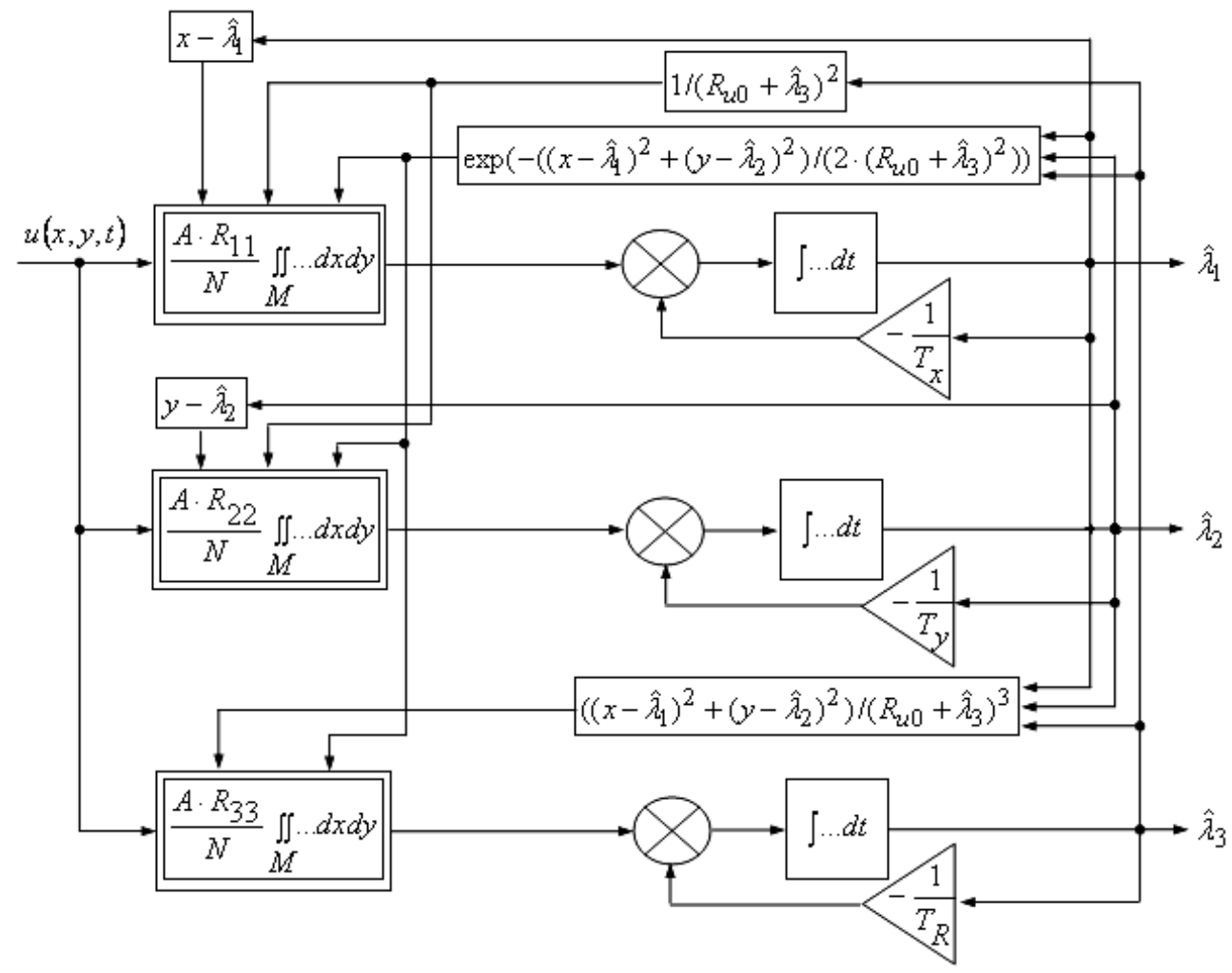

Рис. 3. Структура синтезированного алгоритма

, являющимся оценками координат движущегося объекта. Особенность данной системы заключается в возможности слежения за приращением эквивалентного радиуса изображения факела двигателя $\hat{\lambda}_{3}$ и последующего использования для обработки не только всего наблюдаемого изображения, ограниченного областью $M$, но и части наблюдаемого изображения, ограниченного пространственным стробом с размерами сторон, пропорциональными значению текущего радиуса.

При этом наличие пространственного строба, в пределах которого выполняется обработка, является существенным фактором, определяющим высокую помехозащищенность синтезированного алгоритма от преднамеренных помех.

\section{Исследование эффективности синтезированного алгоритма}

С использованием выражений (14) разработана имитационная модель системы слежения, которая позволила провести оценку точности определения текущих координат движущегося объекта. В качестве показателя эффективности использовалось СКО ошибок измерения оцениваемых текущих параметров полезного сигнала относительно заданных при моделировании.

В качестве изображений фона в разработанной модели применены синтезированные методами компьютерной графики гауссовские случайные поля с заданными параметрами закона распределения яркостей элементов изображения $\left(m_{\phi}=0,5, \sigma_{\phi}=0,1\right)$ и изотропными корреляционными свойствами. 
В цепи отработки ошибок слежения в модели использовались динамические звенья с передаточной функцией разомкнутой петли отработки этих ошибок вида $\frac{K}{p(T p+1)}$. На вход этой передаточной функции с параметрами $K=15$ 1/c, $T=0,03$ с подавались сигналы управления с дискриминаторов $\left(\frac{\partial F(t, \hat{\lambda})}{\partial \hat{\lambda}_{1}}, \frac{\partial F(t, \hat{\lambda})}{\partial \hat{\lambda}_{2}}, \frac{\partial F(t, \hat{\lambda})}{\partial \hat{\lambda}_{3}}\right)$, а с выхода снимались сигналы $\hat{\lambda}_{1}, \hat{\lambda}_{2}, \hat{\lambda}_{3}$. Коэффициенты $R_{11}, R_{22}, R_{33}$ определялись заблаговременно решением стационарной системы обыкновенных дифференциальных уравнений (8). Значение эквивалентного радиуса изображения факела двигателя $R_{u 0}$ составляло 10 элементов разрешения.

В результате имитационного моделирования процесса слежения за координатами объекта, движение которого представлялось как случайный процесс с нулевым математическим ожиданием, среднеквадратическим отклонением порядка 15 элементов разрешения и интервалом временной корреляции $2 \ldots .3 \mathrm{c}$, установлено, что синтезированный алгоритм обеспечивает устойчивое слежение за координатами движущегося объекта с ошибкой не более двух элементов разрешения $\left(\sigma_{0}=2\right)$, что позволяет сделать вывод о его достаточно высокой эффективности.

Кроме того, получены результаты, давшие возможность оценить эффективность функционирования синтезированного алгоритма. На рис. 4 сплошной линией представлен нормированный к $\sigma_{0}$ график зависимости изменения СКО ошибок измерения координат движущегося объекта от отношения реального значения эквивалентного радиуса изображения факела двигателя к априорно заданному $\left(R_{u} / R_{u 0}\right)$ при отсутствии канала слежения за эквивалентным радиусом изображения факела двигателя.

Видно, что алгоритм обеспечивает устойчивое слежение за координатами движущегося объекта с ошибкой не более трех элементов разрешения при условии, что значение реального эквивалентного радиуса отличается от априорно заданного не более чем в 2 раза. В противном случае ошибки слежения значительно возрастают (в 2 раза и более).

Пунктирной линией на рис. 4 представлен нормированный график зависимости изменения СКО ошибок измерения координат объекта от отношения реальной интенсивности изо-

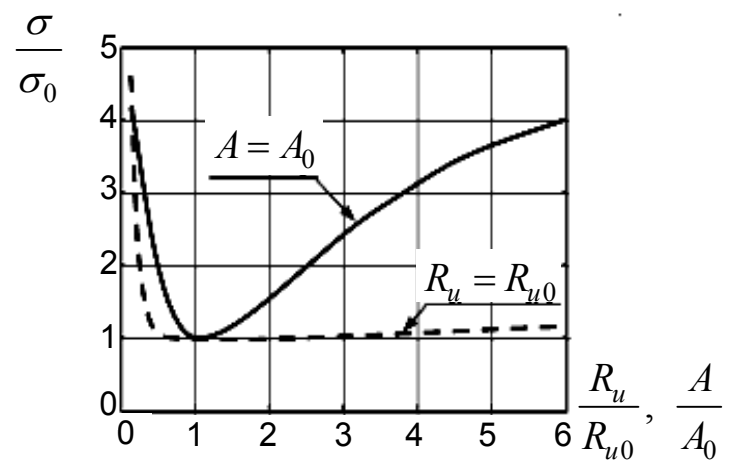

Рис. 4. Нормированные графики зависимости $\sigma / \sigma_{0}$ от $R u / R_{\mathcal{u}}(--)$ и $A / A_{0}(--)$ 
бражения факела двигателя к заданной априори $\left(A / A_{0}\right)$ (при наличии канала слежения за эквивалентным радиусом изображения факела двигателя). Полученные результаты позволяют сделать вывод о том, что несоответствие значений реальной амплитуды полезного сигнала и априорно заданной практически не влияет на качество слежения за текущими координатами движущегося объекта с помощью разработанного алгоритма. Это объясняется тем, что изменения значений реальной амплитуды полезного сигнала приводят к изменению значений оцениваемого эквивалентного радиуса изображения факела двигателя и также косвенно учтены в структуре синтезированного алгоритма.

\section{Заключение}

С использованием аппарата нелинейной пространственно-временной фильтрации синтезирован алгоритм определения координат движущегося объекта по изображению визируемой сцены и факела двигателя управляемого боеприпаса как вторичного источника оптического излучения. Результаты синтеза положены в основу имитационной модели системы слежения, воспроизводящей процесс работы алгоритма и выработки команд в контур высокоточного сопровождения объекта.

Проведена оценка точности слежения за текущими координатами движущегося объекта в условиях отсутствия достоверной информации о параметрах изображения факела двигателя. Полученные результаты говорят об эффективности синтезированного алгоритма и возможности его использования для высокоточного сопровождения объектов с вторичными источниками оптического излучения.

\section{Список литературы}

[1] Баклицкий В.К., Юрьев А.Н. Корреляционно-экстремальные методы навигации. М.: Радио и связь, 1982. 256 с. [Baklitsky V. K., Yuryev A.N. Correlation and Extreme Methods of Navigation. Moscow, Radio and communication, 1982. 256 p. (in Russian)]

[2] Тихонов В.И. Оптимальный прием сигналов. М.: Радио и связь, 1983. 320 с. [Tikhonov V. I. Optimum Reception of Signals. Moscow, Radio and communication, 1983. 320 p. (in Russian)]

[3] Юхно П.М. Траекторно-пространственная фильтрация в корреляционноэкстремальных системах. Автоматика и телемеханика, 1992, 7, 79-86 [Yukhno P.М. А Trajectory and Spatial Filtration in Correlation and Extreme Systems. Automatic Equipment and Telemechanics, 1992, 7, 79-86 (in Russian)]

[4] Козирацкий Ю.Л., Донцов А.А., Козирацкий А.Ю. Прохоров Д.В. и др. Обнаружение и координатометрия оптико-электронных средств, оценка параметров их сигналов. М.: Радиотехника, 2015. 456 c. [Koziratsky Yu.L., Dontsov A.A., Koziratsky A. Yu., Prokhorov D.V. Detection and Koordinatometriya of Optical-electronic Means, Assessment of Parameters of their Signals. Moscow, Radioengineering, 2015, 456 p. (in Russian)]

[5] Донцов А.А., Чернухо И.И. Синтез и анализ алгоритма оценки координат объекта, маркированного оптическим источником излучения. Радиотехника, 2011, 8, 17-21 [Dontsov A.А., Chernucho I.I. Synthesis and the Analysis of Algorithm of an Estimation of Coordinates of Object, Marked by Optical Source Radiation, Radioengineering, 2011, 8, 17-21 (in Russian)] 
[6] Донцов А.А. Синтез алгоритма определения координат движущегося объекта по изображению визируемой сцены. Вестник Воронежского военного института, Воронеж: ВИРЭ, 2007, 1, 87-94 [Dontsov A.A. Synthesis of Algorithm of Determination of Coordinates of Moving Object According to the Image of the Scene. The Bulletin of the Voronezh Military Institute, Voronezh, 2007, 1, 87-94 (in Russian)]

[7] Гарин Е.Н., Ратушняк В.Н. Определение местоположения, взаимного положения и пространственной ориентации подвижных зенитно-ракетных комплексов и средств радиолокации по сигналам СРНС. Успехи современной радиоэлектроники, 2014, 12, 17-20 [Garin E.N., Ratushnyak V.N. Determination of Location, a Mutual Provision and Spatial Orientation of Mobile Surface-to-air Missile Systems and Means of a Radar-location on GPS signals. Achievements of Modern Radio Electronics, 2014, 12, 17-20 (in Russian)] 